PROCEEDINGS OF THE

AMERICAN MATHEMATICAL SOCIETY

Volume 127, Number 3, March 1999, Pages 819-828

S 0002-9939(99)05088-1

\title{
A CHARACTERIZATION OF THE CLIFFORD TORUS
}

\author{
QING-MING CHENG AND SUSUMU ISHIKAWA
}

(Communicated by Christopher Croke)

\begin{abstract}
In this paper, we prove that an $n$-dimensional closed minimal hypersurface $M$ with Ricci curvature $\operatorname{Ric}(M) \geq \frac{n}{2}$ of a unit sphere $S^{n+1}(1)$ is isometric to a Clifford torus if $n \leq S \leq n+\frac{14(n+4)}{9 n+30}$, where $S$ is the squared norm of the second fundamental form of $M$.
\end{abstract}

\section{INTRODUCTION}

Let $M$ be an $n$-dimensional closed minimal hypersurface in a unit sphere $S^{n+1}(1)$ of dimension $n+1$. Let $S$ denote the squared norm of the second fundamental form of $M$. From the Gauss equation (see section 2), we know that $S$, which is extrinsic by definition, is actually an intrinsic quantity. It is well-known that Chern, do Carmo and Kobayashi [3] and Lawson [4] obtained independently that Clifford tori are the only closed minimal hypersurfaces of the unit sphere with $S=n$. When the scalar curvature of $M$ is constant, Yang and the first named author proved in [6] and [7] that if $n \leq S \leq n+\frac{n}{3}$, then $M$ is isometric to a Clifford torus $S^{m}\left(\sqrt{\frac{m}{n}}\right) \times S^{n-m}\left(\sqrt{\frac{n-m}{n}}\right)$. A natural problem is that, for a closed minimal hypersurface $M$ of a unit sphere, whether there exists a constant $\epsilon(n)>0$ such that if $n \leq S \leq n+\epsilon(n)$, then $S=n$ and $M$ is isometric to a Clifford torus $S^{m}\left(\sqrt{\frac{m}{n}}\right) \times S^{n-m}\left(\sqrt{\frac{n-m}{n}}\right)$. The first named author [2] gave a positive answer under the additional condition that $M$ has only two distinct principal curvatures. In general, it still remains open and it is a very hard problem. On the other hand, the Clifford torus $S^{m}\left(\sqrt{\frac{m}{n}}\right) \times S^{n-m}\left(\sqrt{\frac{n-m}{n}}\right)$ is a closed minimal hypersurface in $S^{n+1}(1)$ with $S=n$ and its Ricci curvature varies between $\frac{n(m-1)}{m}$ and $\frac{n(n-m-1)}{n-m}$. If $2 \leq m \leq n-2$, then $\operatorname{Ric}(M) \geq \frac{n}{2}$. Hence it is natural to ask

Received by the editors May 15, 1996 and, in revised form, November 1, 1996.

1991 Mathematics Subject Classification. Primary 53C20, 53C42.

Key words and phrases. Minimal hypersurfaces, scalar curvature, Ricci curvature, Clifford torus.

The first author's research was partially supported by a Grant-in-Aid for Scientific Research from the Japanese Ministry of Education, Science and Culture and by a Grant-in-Aid for Scientific Research from Josai University.

The second author's research was partially supported by a Grant-in-Aid for Scientific Research from the Japanese Ministry of Education, Science and Culture.

(C)1999 American Mathematical Society 
whether there exists a constant $\epsilon(n)>0$ such that if $M$ is a closed minimal hypersurface with $\operatorname{Ric}(M) \geq \frac{n}{2}$ and $n \leq S \leq n+\epsilon(n)$, then $S=n$ and $M$ is isometric to a Clifford torus $S^{m}\left(\sqrt{\frac{m}{n}}\right) \times S^{n-m}\left(\sqrt{\frac{n-m}{n}}\right)(1<m<n-1)$. In this paper, we give an affirmative answer for the above problem.

Theorem 1. Let $M$ be an n-dimensional closed minimal hypersurface of a unit sphere $S^{n+1}(1)$ with Ricci curvature $\operatorname{Ric}(M) \geq \frac{n}{2}$. If

$$
n \leq S \leq n+\frac{14(n+4)}{9 n+30},
$$

then $S=n$ and $M$ is isometric to a Clifford torus $S^{m}\left(\sqrt{\frac{m}{n}}\right) \times S^{n-m}\left(\sqrt{\frac{n-m}{n}}\right)$ $(1<m<n-1)$.

In particular, if $n \leq 5$, we obtain the following

Theorem 2. Let $M$ be an $n$-dimensional $(n \leq 5)$ closed minimal hypersurface of a unit sphere $S^{n+1}(1)$. If

$$
n \leq S \leq n+\epsilon(n),
$$

then $S=n$ and $M$ is isometric to a Clifford torus $S^{m}\left(\sqrt{\frac{m}{n}}\right) \times S^{n-m}\left(\sqrt{\frac{n-m}{n}}\right)$, where $\epsilon(3)=\frac{42}{85}, \epsilon(4)=\frac{8}{31}$ and $\epsilon(5)=\frac{3(21-5 \sqrt{17})}{28+3 \sqrt{17}}$.

Remark. For $n \leq 5$, Peng-Terng [5] proved the following: Let $M$ be an $n$-dimensional $(n \leq 5)$ closed minimal hypersurface of a unit sphere $S^{n+1}(1)$. If

$$
n \leq S \leq n+\epsilon_{1}(n),
$$

then $S=n$, where $\epsilon_{1}(n)=\frac{6-1.13 n}{5+\sqrt{17}}$. It is obvious that our pinching constant in Theorem 2 is larger than theirs.

\section{LOCAL FORMULAE}

Let $M$ be an $n$-dimensional hypersurface in a unit sphere $S^{n+1}(1)$. We choose a local orthonormal frame field $\left\{e_{1}, \ldots, e_{n+1}\right\}$ in $S^{n+1}(1)$, restricted to $M$, so that $e_{1}, \ldots, e_{n}$ are tangent to $M$. Let $\omega_{1}, \ldots, \omega_{n+1}$ denote the dual coframe field in $S^{n+1}(1)$. Then, in $M$,

$$
\omega_{n+1}=0
$$

It follows from Cartan's Lemma that

$$
\omega_{n+1 i}=\sum_{j} h_{i j} \omega_{j}, \quad h_{i j}=h_{j i} .
$$

The second fundamental form $\alpha$ and the mean curvature of $M$ are defined by

$$
\alpha=\sum_{i, j} h_{i j} \omega_{i} \omega_{j} e_{n+1} \quad \text { and } \quad n H=\sum_{i} h_{i i},
$$

respectively. We recall that $M$ is by definition a minimal hypersurface if the mean curvature of $M$ is identically zero. The connection form $\omega_{i j}$ is characterized by the 
structure equations

$$
\left\{\begin{array}{l}
d \omega_{i}+\sum_{j} \omega_{i j} \wedge \omega_{j}=0, \quad \omega_{i j}+\omega_{j i}=0, \\
d \omega_{i j}+\sum_{k} \omega_{i k} \wedge \omega_{k j}=\Omega_{i j}, \\
\Omega_{i j}=\frac{1}{2} \sum_{k, l} R_{i j k l} \omega_{k} \wedge \omega_{l},
\end{array}\right.
$$

where $\Omega_{i j}$ (resp. $R_{i j k l}$ ) denotes the curvature form (resp. the components of the curvature tensor) of $M$. The Gauss equation is given by

$$
R_{i j k l}=\left(\delta_{i k} \delta_{j l}-\delta_{i l} \delta_{j k}\right)+\left(h_{i k} h_{j l}-h_{i l} h_{j k}\right) .
$$

The covariant derivative $\nabla \alpha$ of the second fundamental form $\alpha$ of $M$ with components $h_{i j k}$ is given by

$$
\sum_{k} h_{i j k} \omega_{k}=d h_{i j}+\sum_{k} h_{j k} \omega_{i k}+\sum_{k} h_{i k} \omega_{j k} .
$$

Then the exterior derivative of (2.0) together with the structure equation yields the following Codazzi equation:

$$
h_{i j k}=h_{i k j}=h_{j i k} .
$$

From the Codazzi equation, we know that $h_{i j k}$ is symmetric in the indices $i, j$ and $k$. Similarly, we have the covariant derivative $\nabla^{2} \alpha$ of $\nabla \alpha$ with components $h_{i j k l}$ as follows:

$$
\sum_{l} h_{i j k l} \omega_{l}=d h_{i j k}+\sum_{l} h_{l j k} \omega_{i l}+\sum_{l} h_{i l k} \omega_{j l}+\sum_{l} h_{i j l} \omega_{k l},
$$

and it is easy to get the following Ricci formula:

$$
h_{i j k l}-h_{i j l k}=\sum_{m} h_{i m} R_{m j k l}+\sum_{m} h_{m j} R_{m i k l} .
$$

Similarly, we also have

$$
h_{i j k l m}-h_{i j k m l}=\sum_{r} h_{r j k} R_{r i l m}+\sum_{r} h_{i r k} R_{r j l m}+\sum_{r} h_{i j r} R_{r k l m},
$$

where the $h_{i j k l m}$ 's are the components of the covariant derivative $\nabla^{3} \alpha$ of $\nabla^{2} \alpha$. We should remark that $h_{i j k l}$ and $h_{i j k l m}$ are symmetric in the first three indices $i, j$ and $k$ and generally not symmetric in the other ones. The components of the Ricci curvature and the scalar curvature are given by

$$
\begin{gathered}
R_{i j}=(n-1) \delta_{i j}-\sum_{k} h_{i k} h_{j k}, \\
R=n(n-1)-\sum_{i, j} h_{i j}^{2} .
\end{gathered}
$$

Now we compute certain local formulae. For any fixed point $p$ in $M$, we can choose a local orthonormal frame field $e_{1}, \ldots, e_{n}$ such that

$$
h_{i j}= \begin{cases}0 & \text { if } \quad i \neq j, \\ \lambda_{i} & \text { if } \quad i=j .\end{cases}
$$

The following formulas can be obtained by a direct computation (cf. [1]). Let

$$
S:=\sum_{i, j} h_{i j}^{2}=\sum_{i} \lambda_{i}^{2}
$$




$$
\begin{gathered}
\frac{1}{2} \Delta S=\sum_{i, j, k} h_{i j k}^{2}-S(S-n), \\
\frac{1}{2} \Delta \sum_{i, j, k} h_{i j k}^{2}=\sum_{i, j, k, l} h_{i j k l}^{2}+(2 n+3-S) \sum_{i, j, k} h_{i j k}^{2} \\
+3(2 B-A)-\frac{3}{2}|\nabla S|^{2},
\end{gathered}
$$

where $A=\sum_{i, j, k} \lambda_{i}^{2} h_{i j k}^{2}$ and $B=\sum_{i, j, k} \lambda_{i} \lambda_{j} h_{i j k}^{2}$.

\section{Proofs of the theorems}

At first we give two algebraic lemmas which will play a crucial role in the proofs of our theorems.

Lemma 1. Let $a_{i}(i=1,2,3,4)$ be real numbers satisfying $\sum_{i} a_{i}=0$ and $\sum_{i} a_{i}^{2}=$ a. Then $\sum_{i} a_{i}^{4} \leq \frac{7}{12} a^{2}$.

Proof. We maximize the function $\sum_{i} a_{i}^{4}$ with the constraints $\sum_{i} a_{i}=0$ and $\sum_{i} a_{i}^{2}=$ $a$. By means of the method of the Lagrange multiplier, we solve the following problem:

$$
f=\sum_{i} a_{i}^{4}+\lambda \sum_{i} a_{i}+\mu\left(\sum_{i} a_{i}^{2}-a\right),
$$

where $\lambda$ and $\mu$ are the Lagrange multipliers. The maximum point of $\sum_{i} a_{i}^{4}$ is a critical point of $f$. Taking the derivative of $f$ with respect to $a_{i}$, we have

$$
f_{a_{i}}=4 a_{i}^{3}+\lambda+2 \mu a_{i}=0 .
$$

Hence, at most three of the $a_{i}$ 's are distinct with each other at a critical point of $f$. We consider the following three cases.

(1) Three of the $a_{i}$ 's are distinct with each other. Without loss of generality, we denote them by $a_{1}, a_{2}, a_{3}$ and assume $a_{1}=a_{4}$; then

$$
2 a_{1}+a_{2}+a_{3}=0, \quad 2 a_{1}^{2}+a_{2}^{2}+a_{3}^{2}=a .
$$

Hence,

$$
\begin{aligned}
& \sum_{i} a_{i}^{4}=2 a_{1}^{4}+a_{2}^{4}+a_{3}^{4}=\frac{\left(a+2 a_{1}^{2}\right)^{2}}{2}-14 a_{1}^{4} \\
& =\frac{a^{2}}{2}+2 a a_{1}^{2}-12 a_{1}^{4} \leq \frac{7}{12} a^{2}
\end{aligned}
$$

i.e.,

$$
\sum_{i} a_{i}^{4} \leq \frac{7}{12} a^{2}
$$

(2) Two of the $a_{i}$ 's are distinct with each other. Without loss of generality, we denote them by $a_{1}, a_{2}$ and assume $a_{1}=a_{4}$ and $a_{2}=a_{3}$ or $a_{1}=a_{3}=a_{4}$; then $\sum_{i} a_{i}^{4} \leq \frac{7}{12} a^{2}$.

(3) If all of the $a_{i}$ 's are the same, then $\sum_{i} a_{i}^{4}=0$. 
Therefore, we conclude

$$
\sum_{i} a_{i}^{4} \leq \frac{7}{12} a^{2}
$$

This completes the proof of Lemma 1.

Lemma 2. Let $a_{i j}$ and $b_{i}(i, j=1, \ldots, n)$ be real numbers satisfying $\sum_{i} b_{i}=0$, $\sum_{i} b_{i}^{2}=b>0, \sum_{i, j} b_{i} a_{i j}=\frac{1}{2} b(n-b)$ and $\sum_{i, j} b_{j} a_{i j}=\frac{1}{2} b(n-b)$. Then

$$
\sum_{i} a_{i i}^{2}+3 \sum_{i \neq j} a_{i j}^{2} \geq \frac{3 b(n-b)^{2}}{2(n+4)} .
$$

Proof. We consider $F=\sum_{i} a_{i i}^{2}+3 \sum_{i \neq j} a_{i j}^{2}$ as a function of $a_{i j}$ with constraints $\sum_{i, j} b_{i} a_{i j}=\frac{1}{2} b(n-b)$ and $\sum_{i, j} b_{j} a_{i j}=\frac{1}{2} b(n-b)$. Let

$$
f:=\sum_{i} a_{i i}^{2}+3 \sum_{i \neq j} a_{i j}^{2}+\lambda\left[\sum_{i, j} b_{i} a_{i j}-\frac{1}{2} b(n-b)\right]+\mu\left[\sum_{i, j} b_{j} a_{i j}-\frac{1}{2} b(n-b)\right],
$$

where $\lambda$ and $\mu$ are the Lagrange multipliers. It is obvious that the minimum point of $F$ is a critical point of $f$. Taking the derivative of $f$ with respect to $a_{i j}$, we get

$$
\begin{gathered}
f_{a_{i i}}=2 a_{i i}+\lambda b_{i}+\mu b_{i}=0, \text { for } i, \\
f_{a_{i j}}=6 a_{i j}+\lambda b_{i}+\mu b_{j}=0, \text { for } i \neq j .
\end{gathered}
$$

Hence

$$
\sum_{i} a_{i i} f_{a_{i i}}=2 \sum_{i} a_{i i}^{2}+\lambda \sum_{i} a_{i i} b_{i}+\mu \sum_{i} a_{i i} b_{i}=0
$$

and

$$
\sum_{i \neq j} a_{i j} f_{a_{i j}}=6 \sum_{i \neq j} a_{i j}^{2}+\lambda \sum_{i \neq j} a_{i j} b_{i}+\mu \sum_{i \neq j} a_{i j} b_{j}=0
$$

Therefore,

$$
2\left[\sum_{i} a_{i i}^{2}+3 \sum_{i \neq j} a_{i j}^{2}\right]=\lambda \frac{1}{2} b(b-n)+\mu \frac{1}{2} b(b-n) .
$$

From (3.1) and (3.2), we have

$$
\begin{gathered}
2 \sum_{i} b_{i} a_{i i}+(\lambda+\mu) \sum_{i} b_{i}^{2}=0 \\
6 \sum_{i \neq j} b_{i} a_{i j}+\lambda \sum_{i \neq j} b_{i}^{2}+\mu \sum_{i \neq j} b_{i} b_{j}=0
\end{gathered}
$$

and

$$
6 \sum_{i \neq j} b_{j} a_{i j}+\lambda \sum_{i \neq j} b_{i} b_{j}+\mu \sum_{i \neq j} b_{j}^{2}=0 .
$$

From (3.4) and the two equalities above, we get

$$
-4 \sum_{i} b_{i} a_{i i}+3 b(n-b)+\lambda n b=0
$$


and

$$
\begin{gathered}
-4 \sum_{i} b_{i} a_{i i}+3 b(n-b)+\mu n b=0, \\
\lambda+\mu=\frac{6(b-n)}{(n+4)} .
\end{gathered}
$$

According to (3.3), we obtain

$$
f_{\min }=\frac{3 b(n-b)^{2}}{2(n+4)} \text {. }
$$

Thus we have finished the proof of Lemma 2 .

For any fixed point $p$ in $M$, we can choose a local frame field $e_{1}, \ldots, e_{n}$ such that

$$
h_{i j}=\lambda_{i} \delta_{i j} .
$$

Defining $f_{3}=\sum_{i} \lambda_{i}^{3}$ and $f_{4}=\sum_{i} \lambda_{i}^{4}$, then $f_{3}$ and $f_{4}$ are functions defined globally on $M$.

Proposition 1. Let $M$ be a minimal hypersurface in $S^{n+1}(1)$. Then

$$
\sum_{i, j, k, l} h_{i j k l}^{2} \geq \frac{3}{2}\left(S f_{4}-f_{3}^{2}-2 S^{2}+n S\right)+\frac{3 S(S-n)^{2}}{2(n+4)}
$$

holds.

Proof. From the Ricci formula (2.5) and the Gauss equation (2.3), we have

$$
\begin{aligned}
& h_{i i j j}-h_{j j i i}=h_{i j i j}-h_{i j j i}=\sum_{m} h_{i m} R_{m j i j}+\sum_{m} h_{m j} R_{m i i j} \\
& =\lambda_{i} R_{i j i j}+\lambda_{j} R_{j i i j}=\left(\lambda_{i}-\lambda_{j}\right) R_{i j i j} \\
& =\left(\lambda_{i}-\lambda_{j}\right)\left(1+\lambda_{i} \lambda_{j}\right) .
\end{aligned}
$$

We define

$$
u_{i j k l}=\frac{1}{4}\left(h_{i j k l}+h_{l i j k}+h_{k l i j}+h_{j k l i}\right) .
$$

Since $h_{i j k l}$ is symmetric in the indices $i, j, k$, from formula (3.6), we obtain

$$
\sum_{i, j, k, l} h_{i j k l}^{2} \geq \sum_{i, j, k, l} u_{i j k l}^{2}+\frac{3}{2}\left[S f_{4}-f_{3}^{2}-2 S^{2}+n S\right] .
$$

Since $\Delta h_{i j}=(n-S) h_{i j}$ and $\sum_{i} h_{i i k l}=0$, we have

$$
\sum_{i, j} u_{i i j j} \lambda_{i}=\sum_{i, j} u_{i i j j} \lambda_{j}=\frac{1}{2} S(n-S) .
$$

From $\sum_{i} \lambda_{i}=0$ and $\sum_{i} \lambda_{i}^{2}=S$ and defining $a_{i j}:=u_{i i j j}$ and $b_{i}:=\lambda_{i}$, then $a_{i j}$ and $b_{i}$ satisfy the conditions in Lemma 2 . From the definition of $u_{i j k l}$, we know that $u_{i j k l}$ is symmetric in the indices $i, j, k, l$. From Lemma 2, we infer

$$
\sum_{i, j, k, l} u_{i j k l}^{2} \geq \sum_{i} u_{i i i i}^{2}+3 \sum_{i \neq j} u_{i i j j}^{2} \geq \frac{3 S(S-n)^{2}}{2(n+4)} .
$$


Hence, from (3.8) and (3.9), we obtain

$$
\sum_{i, j, k, l} h_{i j k l}^{2} \geq \frac{3}{2}\left(S f_{4}-f_{3}^{2}-2 S^{2}+n S\right)+\frac{3 S(S-n)^{2}}{2(n+4)} .
$$

This completes the proof of Proposition 1.

Proposition 2. Let $M$ be a closed minimal hypersurface in $S^{n+1}(1)$. Then

$$
\int_{M}\left[\left(S-2 n-\frac{3}{2}\right) \sum_{i, j, k} h_{i j k}^{2}+2(S-n) f_{4}-\frac{3 S(S-n)^{2}}{2(n+4)}+\frac{9}{8}|\nabla S|^{2}\right] d M \geq 0
$$

holds.

Proof. The following integral formula (3.10) can be found in [2]:

$$
\int_{M}(A-2 B) d M=\int_{M}\left[S f_{4}-S^{2}-f_{3}^{2}-\frac{1}{4}|\nabla S|^{2}\right] d M .
$$

From the Ricci formula (2.5), by a direct computation, we obtain

$$
\frac{1}{4} \Delta f_{4}=(n-S) f_{4}+2 A+B .
$$

Integrating both sides of the above equality, we have

$$
\int_{M}(S-n) f_{4} d M=\int_{M}(2 A+B) d M .
$$

Formulas (3.10) and (3.11) yield

$$
\int_{M}\left[(S-4 n) f_{4}+3 f_{3}^{2}+3 S^{2}+\frac{3}{4}|\nabla S|^{2}\right] d M \geq 0
$$

According to Stokes' formula, we integrate the formula (2.11) and obtain

$$
\begin{aligned}
& \int_{M} \sum_{i, j, k, l} h_{i j k l}^{2} d M \\
& =\int_{M}\left[-(2 n+3-S) \sum_{i, j, k} h_{i j k}^{2}-3(2 B-A)+\frac{3}{2}|\nabla S|^{2}\right] d M .
\end{aligned}
$$

From Proposition 1, (3.10) and (3.13), we infer

$$
\begin{aligned}
& \int_{M}\left\{\left(S-2 n-\frac{3}{2}\right) \sum_{i, j, k} h_{i j k}^{2}+\frac{3}{4}|\nabla S|^{2}+\frac{3}{2}\left[S f_{4}-f_{3}^{2}-S^{2}\right]-\frac{3 S(n-S)^{2}}{2(n+4)}\right\} d M \geq 0 . \\
& (3.12)+2 \times(3.14) \text { yields } \\
& \quad \int_{M}\left[\left(S-2 n-\frac{3}{2}\right) \sum_{i, j, k} h_{i j k}^{2}+2(S-n) f_{4}-\frac{3 S(S-n)^{2}}{2(n+4)}+\frac{9}{8}|\nabla S|^{2}\right] d M \geq 0 .
\end{aligned}
$$

Thus Proposition 2 is valid.

Proof of Theorem 1. According to (2.10) and Stokes' Theorem, we obtain

$$
\int_{M} \sum_{i, j, k} h_{i j k}^{2} d M=\int_{M}[S(S-n)] d M
$$


and

$$
-\int_{M} \frac{1}{2}|\nabla S|^{2}=\int_{M}\left[S \sum_{i, j, k} h_{i j k}^{2}+(n-S) S^{2}\right] d M .
$$

From formula (2.7) and the assumption in Theorem 1, we have

$$
R_{i i}=n-1-\lambda_{i}^{2} \geq \frac{n}{2} .
$$

Therefore,

$$
\begin{gathered}
\lambda_{i}^{2} \leq \frac{n-2}{2}, \\
\sum_{i} \lambda_{i}^{4} \leq \frac{n-2}{2} \sum_{i} \lambda_{i}^{2},
\end{gathered}
$$

that is,

$$
f_{4} \leq \frac{n-2}{2} S
$$

From Proposition 2 and (3.17), we have

$$
\int_{M}\left\{\left(S-2 n-\frac{3}{2}\right) \sum_{i, j, k} h_{i j k}^{2}+(n-2) S(S-n)-\frac{3 S(S-n)^{2}}{2(n+4)}+\frac{9}{8}|\nabla S|^{2}\right\} d M \geq 0 .
$$

From (3.15), (3.16) and the above inequality, we infer

$$
\int_{M}\left\{\left(-\frac{5}{4} S-n-\frac{7}{2}\right) \sum_{i, j, k} h_{i j k}^{2}+\left[\frac{9}{4} S-\frac{3(S-n)}{2(n+4)}\right] S(S-n)\right\} d M \geq 0 .
$$

Since

$$
n \leq S \leq n+\frac{14(n+4)}{9 n+30}
$$

we have

$$
\int_{M}\left\{\left(-\frac{5}{4} S-n-\frac{7}{2}\right) \sum_{i, j, k} h_{i j k}^{2}+\left(\frac{9 n}{4}+\frac{7}{2}\right) S(S-n)\right\} d M \geq 0
$$

Hence

$$
\int_{M} \frac{5}{4}(S-n) \sum_{i, j, k} h_{i j k}^{2} d M=0
$$

Since $S$ and $\sum_{i, j, k} h_{i j k}^{2}$ are continuous functions, we have $S=n$. Thus, $M$ is isometric to a Clifford torus $S^{m}\left(\sqrt{\frac{m}{n}}\right) \times S^{n-m}\left(\sqrt{\frac{n-m}{n}}\right)(1<m<n-1)$ from the result due to Chern, do Carmo and Kobayashi [3] or Lawson [4]. This completes the proof of Theorem 1 .

Proof of Theorem 2. In the case $n=3$, because $\sum_{i} \lambda_{i}=0$, we have $f_{4}=\sum_{i} \lambda_{i}^{4}=$ $\frac{S^{2}}{2}$. From Proposition 2, we have

$$
\int_{M}\left\{\left(S-2 n-\frac{3}{2}\right) \sum_{i, j, k} h_{i j k}^{2}+S^{2}(S-n)-\frac{3 S(S-n)^{2}}{2(n+4)}+\frac{9}{8}|\nabla S|^{2}\right\} d M \geq 0 .
$$


From (3.16) and the above inequality, we have

$$
\int_{M}\left\{\left(-\frac{5}{4} S-2 n-\frac{3}{2}\right) \sum_{i, j, k} h_{i j k}^{2}+\frac{13}{4} S^{2}(S-n)-\frac{3 S(S-n)^{2}}{2(n+4)}\right\} d M \geq 0 .
$$

Since

$$
n \leq S \leq n+\frac{42}{85}
$$

we have

$$
\int_{M}\left\{-\frac{5}{4}(S-n) \sum_{i, j, k} h_{i j k}^{2}\right\} d M \geq 0
$$

Hence

$$
\int_{M} \frac{5}{4}(S-n) \sum_{i, j, k} h_{i j k}^{2} d M=0
$$

By making use of the same proof as in Theorem 1, we know that Theorem 2 is true in the case $n=3$.

In the case $n=4$, from Lemma 1 , we have $f_{4} \leq \frac{7}{12} S^{2}$. By using this inequality, we obtain, from Proposition 2,

$$
\int_{M}\left\{\left(S-2 n-\frac{3}{2}\right) \sum_{i, j, k} h_{i j k}^{2}+\frac{7}{6} S^{2}(S-n)-\frac{3 S(S-n)^{2}}{2(n+4)}+\frac{9}{8}|\nabla S|^{2}\right\} d M \geq 0 .
$$

By the same proof as in the case $n=3$, we know that Theorem 2 is also valid in the case $n=4$.

In the case $n=5$, from Proposition 1, (3.10) and (3.13), we have

$$
\int_{M}\left\{\left(S-2 n-\frac{3}{2}\right) \sum_{i, j, k} h_{i j k}^{2}+\frac{3}{2}(A-2 B)-\frac{3 S(S-n)^{2}}{2(n+4)}+\frac{9}{8}|\nabla S|^{2}\right\} d M \geq 0 .
$$

Since

$$
\begin{aligned}
& 3(A-2 B)=\sum_{i, j, k}\left(\lambda_{i}^{2}+\lambda_{j}^{2}+\lambda_{k}^{2}-2 \lambda_{i} \lambda_{j}-2 \lambda_{j} \lambda_{k}-2 \lambda_{k} \lambda_{i}\right) h_{i j k}^{2} \\
& =\sum_{i \neq j \neq k \neq i}\left[2\left(\lambda_{i}^{2}+\lambda_{j}^{2}+\lambda_{k}^{2}\right)-\left(\lambda_{i}+\lambda_{j}+\lambda_{k}\right)^{2}\right] h_{i j k}^{2} \\
& +3 \sum_{i \neq k}\left(\lambda_{k}^{2}-4 \lambda_{i} \lambda_{k}\right) h_{i i k}^{2}-3 \sum_{i} \lambda_{i}^{2} h_{i i i}^{2} \\
& \leq \frac{\sqrt{17}+1}{2} S \sum_{i, j, k} h_{i j k}^{2},
\end{aligned}
$$

by making use of this inequality and (3.18), a similar proof as in the case $n=3$ yields that Theorem 2 is also valid in this case. We have finished the proof of Theorem 2 .

\section{Acknowledgement}

The first named author wishes to thank Professor K. Shiohama for his constant encouragement and help. 


\section{REFERENCES}

1. Cheng, Q.M., The classification of complete hypersurfaces with constant mean curvature of space form of dimension 4, Mem. Fac. Sci. Kyushu Univ. 47 (1993), 79-102. MR 94h:53067; Errata CMP 95:01

2. Cheng, Q.M., The rigidity of Clifford torus $S^{1}\left(\sqrt{\frac{1}{n}}\right) \times S^{n-1}\left(\sqrt{\frac{(n-1)}{n}}\right)$, Comment. Math. Helvetici 71 (1996), 60-69. MR 97a:53094

3. Chern, S.S., do Carmo, M. and Kobayashi, S., Minimal submanifolds of a sphere with second fundamental form of constant length, Functional analysis and related fields, Springer, New York, 1970, pp. 59-75. MR 42:8424

4. Lawson, H.B., Local rigidity theorems for minimal hypersurfaces, Ann. of Math. 89 (1969), 179-185. MR 38:6505

5. Peng, C.K. and Terng, C.L., The scalar curvature of minimal hypersurfaces in spheres, Math. Ann. 266 (1983), 105-113. MR 85c:53099

6. Yang, H.C. and Cheng, Q.M., An estimate of the pinching constant of minimal hypersurfaces with constant scalar curvature in the unit spheres, Manuscripta Math. 84 (1994), 89-100. MR 95c:53076

7. Yang, H.C. and Cheng, Q.M., Chern's conjecture on minimal hypersurfaces, Math. Z. 227 (1998), 377-390. CMP 98:11

Department of Mathematics, Faculty of Science, Josai University, Sakado, Saitama 350-0295, JAPAN

E-mail address: cheng@math.josai.ac.jp

Department of Mathematics, Saga University, Saga 840-0027, Japan 